\title{
On the similarity transformations in second quantized fermion-boson interacting hamiltonian and the BCS theory
}

\author{
Sobre a transformação de similaridade em sistemas férmion-bóson interagentes na segunda \\ quantização e a teoria BCS
}

C.A. Dartora, Fillipi Klos Rodrigues de Campos

Departamento de Engenharia Elétrica, Universidade Federal do Paraná, PR, Brasil

Received on December 28, 2015. Revised on April 4, 2016. Accepted on April 14, 2016

\begin{abstract}
Uma transformação de similaridade é uma relação de equivalência entre matrizes quadradas que preserva o determinante, traço e autovalores da matriz, tendo papel fundamental na mecânica quântica para fins de simplificar sistemas hamiltonianos muito complexos, o que permite melhorar de forma significativa as soluções analíticas no uso de teoria de perturbações. Um exemplo prototípico desse tipo é a teoria BCS para a supercondutividade convencional, derivada de uma transformação de similaridade da interação elétron-fônon original, escrita na forma de segunda quantização. Nesse trabalho, discutimos o método geral para escrever o operador de transformação de similaridade na forma de segunda quantização, permitindo reescrever o hamiltoniano de interação entre férmions e bósons, obtendo uma teoria efetiva em que somente os graus de liberdade de interesse são retidos depois da transformação.

Palavras-chave: sistemas férmion-bóson, transformações de similaridade, segunda quantização, supercondutividade.
\end{abstract}

A similarity transformation is an equivalence relation between square matrices which preserves determinant, trace and eigenvalues, playing a key role in quantum mechanics in simplifying complex hamiltonian systems and improving analytical results attainable from the use of perturbation theory. As a prototypical example, the conventional BCS theory of superconductivity is usually derived from a similarity transformation of the original electron-phonon hamiltonian, written in second quantized version. Here we discuss the general method for writing the similarity transformation operator in second quantized form, allowing one to recast a hamiltonian describing an interacting fermion-boson system into an effective theory in which only the desired degrees of freedom are kept after the transformation. Keywords: fermion-boson systems; similarity transformation; second quantization; superconductivity.

\section{Introduction}

There is little room for controversy in stating that fermion-boson interacting systems are among the most general problems in physics, since they can be used to describe almost everything in the real world, at least at a quantum-mechanical level. For instance, in the standard model of elementary particles the interaction between fermions(electrons, neutrinos and quarks) are mediated by the so-called gauge bosons (photons, gluons and so on) [1-3]. In cavity quantum electrodynamics problems, fermion states

*Endereço de correspondência: cadartora@eletrica.ufpr.br in a multilevel system are coupled via photons, leading to a large class of problems that can be mapped to the Jaynes-Cummings models [4,5]. Electronic transport properties are affected by electron-phonon coupling in solids, leading to the phenomenon of superconductivity in many materials [6 6 ]. Despite the apparent simplicity of fermion-boson models when written in second quantization formalism, the vast majority of these problems are not solvable in an exact manner and, therefore, demand clever methods and mathematical tricks in order to obtain an analytically tractable perturbation scheme, allowing one to predict meaningful physical results. 
As a matter of fact, the term second quantization is synonymous to quantum field theory, which naturally describes many-body problems and can be represented in two very dissimilar but otherwise equivalent ways, to be known $[1,-3,9,10]$ : i) Feynman path integral formulation, based on "sum over histories", which is beyond the scope of the present contribution and ii) canonical quantization formalism, firmly grounded on the use of the socalled creation and annihilation operator algebra. While Feynman formalism deals with gaussian integration gymnastics [10], one of the most important mathematical tool for the canonical formalism is the similarity transformation of the hamiltonian. A similarity transformation is an equivalence relation between square matrices which preserves all the relevant information in quantum mechanics, i.e., matrix determinant, trace and eigenvalues. It can be used to promote a change of basis or a symmetry transformation [11, 12], leading to an equivalent hamiltonian, in which the terms too complicated to be dealt with are completely eliminated, at least in first order of a perturbation series expansion, thusly simplifying the problem. In the canonical formalism, the conventional BCS theory of superconductivity is usually derived from a similarity transformation of the original electron-phonon hamiltonian 12 .

In the present contribution, we discuss the general method for writing similarity transformation operators in second quantized form, allowing one to recast a hamiltonian describing an interacting fermionboson system into an effective theory, in which only the desired degrees of freedom are kept after the transformation. The proposed rule of thumb is applied to the problem of electron-phonon interactions in obtaining the BCS theory of superconductivity.

The content of this paper is organized in the following way: in the next Section we will introduce and briefly discuss the fermion-boson general problem in the canonical formalism. In Section 3 the general method for obtaining similarity transformation operators in second-quantized version is presented. Section 4 brings the application of the method to the BCS theory of superconductivity and, finally, in the last Section a few conclusions and remarks are added.

\section{The general form of fermion-boson interacting hamiltonians}

Our primary interest is in the problem of fermionboson interacting systems, described by the follow- ing general hamiltonian:

$$
\begin{aligned}
\hat{H}= & \sum_{i} E_{i} \hat{c}_{i}^{\dagger} \hat{c}_{i}+\sum_{n} \hbar \omega_{n} \hat{a}_{n}^{\dagger} \hat{a}_{n}+ \\
& \sum_{i j n}\left[\gamma_{i j n} \hat{c}_{i}^{\dagger} \hat{c}_{j} \hat{a}_{n}+\gamma_{i j n}^{*} \hat{c}_{j}^{\dagger} \hat{c}_{i} \hat{a}_{n}^{\dagger}\right],
\end{aligned}
$$

where $E_{i}$ are the energy eigenstates of the fermionic degrees of freedom with a set of quantum numbers labeled by the index $i, \hbar \omega_{n}$ is the energy of a bosonic state with quantum numbers labeled by $n$ and $\gamma_{i j n}$ is the fermion-boson coupling parameter, depending on the bosonic quantum number, $\hat{c}_{i}\left(\hat{c}_{i}^{\dagger}\right)$ is a fermionic operator which annihilates (creates) a fermion in the $i$-th quantum state, while $\hat{a}_{i}\left(\hat{a}_{i}^{\dagger}\right)$ is a bosonic annihilation (creation) operator. Fermionic operators must obey the following anti-commuting algebra:

$$
\begin{array}{r}
\left\{\hat{c}_{i}, \hat{c}_{j}\right\}=\left\{\hat{c}_{i}^{\dagger}, \hat{c}_{j}^{\dagger}\right\}=0, \\
\left\{\hat{c}_{i}, \hat{c}_{j}^{\dagger}\right\}=\delta_{i j},
\end{array}
$$

where $\{A, B\}=A B+B A$ is the anti-commutator for any two operators $A$ and $B, \delta_{i j}$ is the Kronecker delta function, yielding $1(0)$ for $i=j(i \neq j)$. By contrast, bosonic operators obey a commuting algebra, explicitly written below:

$$
\begin{array}{r}
{\left[\hat{a}_{i}, \hat{a}_{j}\right]=\left[\hat{a}_{i}^{\dagger}, \hat{a}_{j}^{\dagger}\right]=0,} \\
{\left[\hat{a}_{i}, \hat{a}_{j}^{\dagger}\right]=\delta_{i j},}
\end{array}
$$

where $[A, B]=A B-B A$ is the commutator of $A$ and $B$. The number operators are defined as $\hat{c}_{i}^{\dagger} \hat{c}_{i}$ and $\hat{a}_{n}^{\dagger} \hat{a}_{n}$ for fermions and bosons, respectively.

In the above hamiltonian, the first term, $\sum_{i} E_{i} \hat{c}_{i}^{\dagger} \hat{c}_{i}$, is related to the fermion system alone, whose energies are supposed to be exactly known. The second term, $\sum_{n} \hbar \omega_{n} \hat{a}_{n}^{\dagger} \hat{a}_{n}$, is related to the energy of the bosonic degrees of freedom, and it conserves the boson number, since it is diagonal in the number representation. The last terms represent the fermion-boson interaction, which permits non-conservation of the boson number. For instance, the term $\gamma_{i j n} \hat{c}_{i}^{\dagger} \hat{c}_{j} \hat{a}_{n}$ represents the transition of the fermion from the $j$-th state to the $i$-th state by absorbing a boson with quantum number $n$. The last term $\gamma_{i j n}^{*} \hat{c}_{j}^{\dagger} \hat{c}_{i} \hat{a}_{n}^{\dagger}$ represents the fermion transition with boson emission. In either case, it is clear that the total boson number is not preserved, i.e., states with distinct boson number are coupled due to the interaction. It is usually the case that 
the fermion-boson interaction is weak and can be treated as a small perturbation, which is known as the weak coupling limit.

\section{The general method of similarity transformation}

As the starting point, consider the following hamiltonian:

$$
\hat{H}=\hat{H}_{0}+\lambda \hat{V},
$$

where $\hat{H}_{0}$ is an unperturbed hamiltonian and $\hat{V}$ is the perturbation, usually responsible for the interactions. The parameter $\lambda$ is used to control the intensity of the perturbation term. For $\lambda \rightarrow 0$ interactions are absent, but for small $\lambda$ perturbation theory is generally valid, while for $\lambda \rightarrow \infty$ the system is dominated by the interaction term and perturbation theory breaks down. In order to solve the problem in the weak coupling limit $(\lambda<1)$, we assume the eigenstates of $\hat{H}_{0}$ are known, and therefore we want to eliminate the perturbation term $\hat{V}$, at least to first order in the coupling parameter $\lambda$. This way, in the transformed hamiltonian the perturbation will appear only at the next leading order, which is $\lambda^{2}$. If necessary, the next leading order can also be eliminated by continuing the transformation procedure to be described below. Elimination of $\hat{V}$ to first order in $\lambda$ is accomplished by applying a similarity transformation to $\hat{H}$, as follows:

$$
\hat{H}^{\prime}=e^{\hat{S}} \hat{H} e^{-\hat{S}} .
$$

Notice that, since the hamiltonian must be hermitian in quantum mechanics and $e^{\hat{S}}$ must be an unitary operator, the matrix $\hat{S}$ must obey the relation $\hat{S}^{\dagger}=-\hat{S}$. Expanding the above equation in Taylor series yields:

$\hat{H}^{\prime}=\left(1+\hat{S}+\frac{1}{2 !} \hat{S}^{2}+\ldots\right) \hat{H}\left(1-\hat{S}+\frac{1}{2 !} \hat{S}^{2}+\ldots\right)$,

which can be promptly recast into the following form:

$$
\hat{H}^{\prime}=\hat{H}+[\hat{S}, \hat{H}]+\frac{1}{2}[\hat{S},[\hat{S}, \hat{H}]]+\ldots
$$

After inserting (6) into the above equation, we get:

$$
\begin{aligned}
\hat{H}^{\prime}= & \hat{H}_{0}+\lambda \hat{V}+\left[\hat{S}, \hat{H}_{0}\right]+\lambda[\hat{S}, \hat{V}]+ \\
& \frac{1}{2}\left[\hat{S},\left[\hat{S}, \hat{H}_{0}\right]\right]+\lambda[\hat{S},[\hat{S}, \hat{V}]] \ldots
\end{aligned}
$$

Going further, the term $\lambda \hat{V}$ can be eliminated from the problem to first order in $\lambda$ by making:

$$
\lambda \hat{V}+\left[\hat{S}, \hat{H}_{0}\right]=0 .
$$

Looking at the above equation, it is clear that $\hat{S}$ will depend linearly on $\lambda$. Using the set of $\hat{H}_{0}$ eigenstates, satisfying the eigenvalue equation below,

$$
\hat{H}_{0}|m\rangle=E_{m}|m\rangle,
$$

we can straightforwardly solve the equation (11) for the operator $\hat{S}$, being the result:

$$
\hat{S}=\lambda \sum_{m, n} \frac{\langle m|\hat{V}| n\rangle}{E_{m}-E_{n}}|m\rangle\langle n| .
$$

Making use of (11), we can rewrite the transformed hamiltonian $\hat{H}^{\prime}$ in the following way:

$$
\hat{H}^{\prime}=\hat{H}_{0}+\frac{\lambda}{2}[\hat{S}, \hat{V}]+\ldots
$$

Now the perturbation is in the term $\frac{\lambda}{2}[\hat{S}, \hat{V}]$, which depends on $\lambda^{2}$. It must be pointed out that the quantum state of $\hat{H}$ satisfying the equation $\hat{H}|\psi\rangle=E|\psi\rangle$ is also transformed, $\left|\psi^{\prime}\right\rangle=e^{\hat{S}}|\psi\rangle$, but it possesses the same energy eigenvalue $E$.

Next, we turn our attention to the form of the transformation matrix $\hat{S}$ given by equation 13 . and its connection to the fermion-boson problem. The term $|m\rangle\langle n|$ can be interpreted as an operator which annihilates the initial state $|n\rangle$ and creates the final state $|m\rangle$. It is weighted by the matrix element $\langle m|\hat{V}| n\rangle$ of the perturbation operator $\hat{V}$ divided by the energy difference $\left(E_{m}-E_{n}\right)$ between final and initial states. It is our aim to write $\hat{S}$ as a second-quantized operator, observing the form of the perturbation operator $\hat{V}$. To do that, we must replace $|m\rangle\langle n|$ in (13) by creation and annihilation operators exactly in the same order as they appear in the operator $\hat{V}$. The energy difference $E_{m}-E_{n}$ can be easily calculated considering the total number of particles appearing in the final and initial states. Let us consider the fermion-boson hamiltonian (1) written as follows:

$$
\begin{aligned}
\hat{H}_{0} & =\sum_{i} E_{i} \hat{c}_{i}^{\dagger} \hat{c}_{i}+\sum_{n} \hbar \omega_{n} \hat{a}_{n}^{\dagger} \hat{a}_{n}, \\
\lambda \hat{V} & =\sum_{i j n}\left[\gamma_{i j n} \hat{c}_{i}^{\dagger} \hat{c}_{j} \hat{a}_{n}+\gamma_{i j n}^{*} \hat{c}_{j}^{\dagger} \hat{c}_{i} \hat{a}_{n}^{\dagger}\right] .
\end{aligned}
$$

Taking a closer look at the term $\gamma_{i j n} \hat{c}_{i}^{\dagger} \hat{c}_{j} \hat{a}_{n}$ in $\hat{V}$, we know that it will annihilate a boson in the $n$-th state 
and a fermion in the $j$-th state, creating a fermion in the $i$-th final state. Therefore, the initial energy of the fermion-boson system is $E_{0}+E_{j}+\hbar \omega_{n}$ and the final energy is $E_{0}+E_{i}$, where $E_{0}$ is the sum of all other energies in the system, not involved in this specific interaction process. The energy difference between final and initial states will be given by $E_{i}-$ $E_{j}-\hbar \omega_{n}$. In writing down the matrix $\hat{S}$, this term will show up as $\gamma_{n} \hat{c}_{i}^{\dagger} \hat{c}_{j} \hat{a}_{n} /\left(E_{i}-E_{j}-\hbar \omega_{n}\right)$. Taking into consideration the above discussion, we prescribe, as a rule of thumb, a direct method for obtaining $\hat{S}$, as follows: i) write down the perturbation operator $\hat{V}$ for $\hat{S}$ and ii) divide each term by the energy difference between final and initial states produced by that specific term. It is easy to see that for the perturbation 16 the transformation matrix $\hat{S}$ will be given by:

$$
\hat{S}=\sum_{i j n}\left[\frac{\gamma_{i j n} \hat{c}_{i}^{\dagger} \hat{c}_{j} \hat{a}_{n}}{E_{i}-E_{j}-\hbar \omega_{n}}+\frac{\gamma_{i j n}^{*} \hat{c}_{j}^{\dagger} \hat{c}_{i} \hat{a}_{n}^{\dagger}}{E_{j}-E_{i}+\hbar \omega_{n}}\right]
$$

The interested reader is encouraged to demonstrate explicitly that the above expression for $\hat{S}$ exactly solves equation (11), provided the knowledge of the fermionic and bosonic relations (2)-(5) and the Hamiltonian (6), conveniently splitted as (15) and (16).

Notice that the prescribed method for writing down $\hat{S}$ works for very general situations. For the sake of simplicity, consider the boson hamiltonian below:

$$
\hat{H}=\hbar \omega \hat{a}^{\dagger} \hat{a}+\gamma\left(\hat{a}+\hat{a}^{\dagger}\right)
$$

Indeed, the above problem is a trivial one, since it can be exactly diagonalized by the replacement $\hat{a} \rightarrow \hat{a}-\gamma / \hbar \omega$, leading to $\hat{H}=\hbar \omega \hat{a}^{\dagger} \hat{a}-\gamma^{2} /(\hbar \omega)$, but we will use the similarity transformation method in order to eliminate the perturbation $\hat{V}=\gamma(\hat{a}+$ $\left.\hat{a}^{\dagger}\right)$. Following the above mentioned prescription, we write down the matrix $\hat{S}$ :

$$
\hat{S}=-\frac{\gamma}{\hbar \omega} \hat{a}+\frac{\gamma}{\hbar \omega} \hat{a}^{\dagger} .
$$

It is straightforward to show that the energy difference between final and initial state due to the term $\gamma \hat{a}$ is $-\hbar \omega$, since initially there are $n$ bosons of energy $\hbar \omega$ and, after annihilation of one boson we are left with $n-1$ bosons, leading to an energy deficit of $\hbar \omega$. Inserting (18) and (19) into (14) and making use of $\left[\hat{a}, \hat{a}^{\dagger}\right]=1$ we obtain:

$$
\begin{aligned}
\hat{H}^{\prime} & =\hat{H}_{0}+\frac{1}{2}[\hat{S}, \hat{V}] \\
& =\hbar \omega \hat{a}^{\dagger} \hat{a}+\frac{\gamma^{2}}{2 \hbar \omega}\left(-\left[\hat{a}, \hat{a}^{\dagger}\right]+\left[\hat{a}^{\dagger}, \hat{a}\right]\right) \\
& =\hbar \omega \hat{a}^{\dagger} \hat{a}-\frac{\gamma^{2}}{\hbar \omega}
\end{aligned}
$$

which is the exact result in this particular case.

In the next Section we will use the method described here to obtain the BCS hamiltonian describing conventional superconductivity.

\section{The conventional BCS superconductivity theory}

The BCS theory successfully explains the conventional superconductivity of materials, by considering attractive electron-electron interactions mediated by the exchange of virtual phonons(the quantized excitations of lattice vibrations), which leads to the formation of the so-called Cooper pairs, a bound state of electrons in momentum space. The starting point of the BCS theory is the electron-phonon Hamiltonian 12]:

$$
\begin{aligned}
\hat{H}_{0} & =\sum_{\mathbf{k}} E_{\mathbf{k}} \hat{c}_{\mathbf{k}}^{\dagger} \hat{c}_{\mathbf{k}}+\sum_{\mathbf{q}} \hbar \omega_{\mathbf{q}} \hat{a}_{\mathbf{q}}^{\dagger} \hat{a}_{\mathbf{q}}, \\
\lambda \hat{V} & =i \sum_{\mathbf{k q}} D_{\mathbf{q}} \hat{c}_{\mathbf{k}+\mathbf{q}}^{\dagger} \hat{c}_{\mathbf{k}}\left[\hat{a}_{\mathbf{q}}-\hat{a}_{-\mathbf{q}}^{\dagger}\right] .
\end{aligned}
$$

where $\hat{c}_{\mathbf{k}}\left(\hat{c}_{\mathbf{k}}^{\dagger}\right)$ annihilates(creates) an electron(fermion) with momentum $\hbar \mathbf{k}$ (and spin $\sigma=\uparrow, \downarrow$, which is being omitted) and energy $E_{\mathbf{k}}=\hbar^{2} \mathbf{k}^{2} /(2 m), \hat{a}_{\mathbf{q}}\left(\hat{a}_{\mathbf{q}}^{\dagger}\right)$ annihilates (creates) a phonon (boson) with momentum $\hbar \mathbf{q}$ and energy $\hbar \omega_{\mathbf{q}}$, the parameter $D_{\mathbf{q}}$ is the electron-phonon coupling and depends only on the phonon momentum $\hbar \mathbf{q}$, not on the electronic momentum $\hbar \mathbf{k}$, in the present case. It is left as an exercise to show that the requirement of hermiticity of the hamiltonian leads to the condition $D_{\mathbf{q}}^{*}=D_{-\mathbf{q}}$. Using the prescription described in the previous Section we can easily write down the transformation matrix $\hat{S}$ :

$$
\begin{aligned}
\hat{S} & =i \sum_{\mathbf{k q}} D_{\mathbf{q}}\left[\frac{\hat{c}_{\mathbf{k}+\mathbf{q}}^{\dagger} \hat{c}_{\mathbf{k}} \hat{a}_{\mathbf{q}}}{E_{\mathbf{k}+\mathbf{q}}-E_{\mathbf{k}}-\hbar \omega_{\mathbf{q}}}\right. \\
& \left.-\frac{\hat{c}_{\mathbf{k}+\mathbf{q}}^{\dagger} \hat{c}_{\mathbf{k}} \hat{a}_{-\mathbf{q}}^{\dagger}}{E_{\mathbf{k}+\mathbf{q}}-E_{\mathbf{k}}+\hbar \omega_{\mathbf{q}}}\right],
\end{aligned}
$$


where we have used the fact that $\omega_{\mathbf{q}}=\omega_{-\mathbf{q}}$. Going further, we need to determine $[\hat{S}, \lambda \hat{V}]$ in order to obtain $\hat{H}^{\prime}$. The result is:

$$
[\hat{S}, \lambda \hat{V}]=-\sum_{\mathbf{k q}} \sum_{\mathbf{k}^{\prime} \mathbf{q}^{\prime}} D_{\mathbf{q}} D_{\mathbf{q}^{\prime}}\left(A_{1}-A_{2}-A_{3}+A_{4}\right),
$$

where we defined the terms below

$$
\begin{aligned}
& A_{1}=\frac{\left[\hat{c}_{\mathbf{k}+\mathbf{q}}^{\dagger} \hat{c}_{\mathbf{k}} \hat{a}_{\mathbf{q}}, \hat{c}_{\mathbf{k}^{\prime}+\mathbf{q}^{\prime}}^{\dagger} \hat{c}_{\mathbf{k}^{\prime}} \hat{a}_{\mathbf{q}^{\prime}}\right]}{E_{\mathbf{k}+\mathbf{q}}-E_{\mathbf{k}}-\hbar \omega_{\mathbf{q}}}, \\
& A_{2}=\frac{\left[\hat{c}_{\mathbf{k}+\mathbf{q}}^{\dagger} \hat{c}_{\mathbf{k}} \hat{a}_{\mathbf{q}}, \hat{c}_{\mathbf{k}^{\prime}+\mathbf{q}^{\prime}}^{\dagger} \hat{c}_{\mathbf{k}^{\prime}} \hat{a}_{-\mathbf{q}^{\prime}}^{\dagger}\right]}{E_{\mathbf{k}+\mathbf{q}}-E_{\mathbf{k}}-\hbar \omega_{\mathbf{q}}}, \\
& A_{3}=\frac{\left[\hat{c}_{\mathbf{k}+\mathbf{q}}^{\dagger} \hat{c}_{\mathbf{k}} \hat{a}_{-\mathbf{q}}^{\dagger}, \hat{c}_{\mathbf{k}^{\prime}+\mathbf{q}^{\prime}}^{\dagger} \hat{c}_{\mathbf{k}^{\prime}} \hat{a}_{\mathbf{q}^{\prime}}\right]}{E_{\mathbf{k}+\mathbf{q}}-E_{\mathbf{k}}+\hbar \omega_{\mathbf{q}}}, \\
& A_{4}=\frac{\left[\hat{c}_{\mathbf{k}+\mathbf{q}}^{\dagger} \hat{c}_{\mathbf{k}} \hat{a}_{-\mathbf{q}}^{\dagger}, \hat{c}_{\mathbf{k}^{\prime}+\mathbf{q}^{\prime}}^{\dagger} \hat{c}_{\mathbf{k}^{\prime}} \hat{a}_{-\mathbf{q}^{\prime}}^{\dagger}\right]}{E_{\mathbf{k}+\mathbf{q}}-E_{\mathbf{k}}+\hbar \omega_{\mathbf{q}}} .
\end{aligned}
$$

As a final step, we want to retain only fermionic degrees of freedom by tracing out the phonon variables. It can be done by averaging phonon operators in the canonical ensemble, for which the density matrix is written as $\hat{\rho}=e^{-\beta \sum_{\mathbf{q}} \hbar \omega_{\mathbf{q}} \hat{a}_{\mathbf{q}}^{\dagger} \hat{a}_{\mathbf{q}}} / \operatorname{tr}\left(e^{-\beta \sum_{\mathbf{q}} \hbar \omega_{\mathbf{q}} \hat{a}_{\mathbf{q}}^{\dagger} \hat{a}_{\mathbf{q}}}\right)$, where $\beta=1 /\left(k_{B} T\right)$ is the reciprocal of the temperature. Notice that the terms $A_{1}$ and $A_{4}$ vanish by virtue of the trace properties $\operatorname{tr}\left(\hat{\rho} \hat{a}_{\mathbf{q}} \hat{a}_{\mathbf{q}^{\prime}}\right)=$ $\operatorname{tr}\left(\hat{\rho} \hat{a}_{\mathbf{q}}^{\dagger} \hat{a}_{\mathbf{q}^{\prime}}^{\dagger}\right)=0$. The remaining terms must be carefully calculated, taking into account that in the canonical ensemble $\operatorname{tr}\left(\hat{\rho} \hat{a}_{\mathbf{q}}^{\dagger} \hat{a}_{\mathbf{q}^{\prime}}\right)=\hat{n}_{\mathbf{q}} \delta_{\mathbf{q}, \mathbf{q}^{\prime}}$ for phonon operators. The commutators appearing in $A_{3}$ and $A_{4}$ are of the form below:

$\left[\hat{c}_{i}^{\dagger} \hat{c}_{j} \hat{a}_{m}, \hat{c}_{k}^{\dagger} \hat{c}_{l} \hat{a}_{n}^{\dagger}\right]=\delta_{m, n} \hat{c}_{i}^{\dagger} \hat{c}_{j} \hat{c}_{k}^{\dagger} \hat{c}_{l}+\hat{a}_{n}^{\dagger} \hat{a}_{m}\left[\hat{c}_{i}^{\dagger} \hat{c}_{j}, \hat{c}_{k}^{\dagger} \hat{c}_{l}\right]$,

where $i=\mathbf{k}+\mathbf{q}, j=\mathbf{k}$ and so on. The commutator $\left[\hat{c}_{i}^{\dagger} \hat{c}_{j}, \hat{c}_{k}^{\dagger} \hat{c}_{l}\right]$ can be handled with the help of the following identity:

$$
\begin{aligned}
{[A B, C D]=} & A\{B, C\} D-\{A, C\} B D+ \\
& C A\{B, D\}-C\{A, D\} B .
\end{aligned}
$$

The last steps towards the BCS hamiltonian are left as an exercise, but the result $\hat{H}^{\prime}=\hat{H}_{0}+\frac{1}{2}[\hat{S}, \lambda \hat{V}]$, after averaging over the phonon operators, is shown below:

$$
\begin{aligned}
& \hat{H}^{\prime}=\sum_{\mathbf{k}} E_{\mathbf{k}}^{\prime} c_{\mathbf{k}}^{\dagger} c_{\mathbf{k}}+\frac{1}{2} \sum_{\mathbf{k} \mathbf{k}^{\prime} \mathbf{q}}\left|D_{\mathbf{q}}\right|^{2} \\
& \times\left[\frac{\hat{c}_{\mathbf{k}+\mathbf{q}}^{\dagger} \hat{c}_{\mathbf{k}} \hat{c}_{\mathbf{k}^{\prime}-\mathbf{q}}^{\dagger} \hat{c}_{\mathbf{k}^{\prime}}}{E_{\mathbf{k}+\mathbf{q}}-E_{\mathbf{k}}-\hbar \omega_{\mathbf{q}}}-\frac{\hat{c}_{\mathbf{k}^{\prime}-\mathbf{q}}^{\dagger} \hat{c}_{\mathbf{k}^{\prime}} \hat{c}_{\mathbf{k}+\mathbf{q}}^{\dagger} \hat{c}_{\mathbf{k}}}{E_{\mathbf{k}+\mathbf{q}}-E_{\mathbf{k}}+\hbar \omega_{\mathbf{q}}}\right]
\end{aligned}
$$

where $E_{\mathbf{k}}^{\prime}$ is the electron energy, corrected by phonon interactions:

$$
E_{\mathbf{k}}^{\prime}=E_{\mathbf{k}}-2 \sum_{\mathbf{q}} \frac{n_{\mathbf{q}}\left|D_{\mathbf{q}}\right|^{2}\left(E_{\mathbf{k}+\mathbf{q}}-E_{\mathbf{k}}\right)}{\left(E_{\mathbf{k}+\mathbf{q}}-E_{\mathbf{k}}\right)^{2}-\left(\hbar \omega_{\mathbf{q}}\right)^{2}},
$$

$n_{\mathbf{q}}=\operatorname{tr}\left(\hat{\rho}_{\mathbf{q}} \hat{a}_{\mathbf{q}}^{\dagger} \hat{a}_{\mathbf{q}}\right)=1 /\left(e^{\beta \hbar \omega_{\mathbf{q}}}-1\right)$ is the phonon number. At $T=0$ the phonon number vanishes for any $\mathbf{q}$ and the electron-phonon interactions does not produce any effect on the electron energy, i.e., $E_{\mathbf{k}}^{\prime}=E_{\mathbf{k}}$, but for higher temperatures it deviates from the parabolic energy-momentum dispersion relation, which is characteristic of non-relativistic free particles. Now we will put the effective electronelectron interaction, by making some rearrangements in the last term in (31). Using the fermionic relations one can rewrite $\hat{c}_{\mathbf{k}^{\prime}-\mathbf{q}}^{\dagger} \hat{c}_{\mathbf{k}^{\prime}} \hat{c}_{\mathbf{k}+\mathbf{q}}^{\dagger} \hat{c}_{\mathbf{k}}$ as follows:

$$
\begin{aligned}
& \hat{c}_{\mathbf{k}^{\prime}-\mathbf{q}}^{\dagger} \hat{c}_{\mathbf{k}^{\prime}} \hat{c}_{\mathbf{k}+\mathbf{q}}^{\dagger} \hat{c}_{\mathbf{k}}=\hat{c}_{\mathbf{k}^{\prime}-\mathbf{q}}^{\dagger}\left(\delta_{\mathbf{k}^{\prime}, \mathbf{k}+\mathbf{q}}-\hat{c}_{\mathbf{k}+\mathbf{q}}^{\dagger} \hat{c}_{\mathbf{k}^{\prime}}\right) \hat{c}_{\mathbf{k}}, \\
& =\delta_{\mathbf{k}^{\prime}, \mathbf{k}+\mathbf{q}} \hat{c}_{\mathbf{k}^{\prime}-\mathbf{q}}^{\dagger} \hat{c}_{\mathbf{k}}-\hat{c}_{\mathbf{k}+\mathbf{q}}^{\dagger} \hat{c}_{\mathbf{k}^{\prime}-\mathbf{q}}^{\dagger} \hat{c}_{\mathbf{k}} \hat{c}_{\mathbf{k}^{\prime}} \\
& =\delta_{\mathbf{k}^{\prime}, \mathbf{k}+\mathbf{q}} \hat{c}_{\mathbf{k}^{\prime}-\mathbf{q}}^{\dagger} \hat{c}_{\mathbf{k}}-\hat{c}_{\mathbf{k}+\mathbf{q}}^{\dagger}\left(\delta_{\mathbf{k}, \mathbf{k}^{\prime}-\mathbf{q}}-\hat{c}_{\mathbf{k}} \hat{c}_{\mathbf{k}^{\prime}-\mathbf{q}}^{\dagger}\right) \hat{c}_{\mathbf{k}^{\prime}} \\
& =\delta_{\mathbf{k}^{\prime}, \mathbf{k}+\mathbf{q}} \hat{c}_{\mathbf{k}^{\prime}-\mathbf{q}}^{\dagger} \hat{c}_{\mathbf{k}}-\delta_{\mathbf{k}, \mathbf{k}^{\prime}-\mathbf{q}} \hat{c}_{\mathbf{k}+\mathbf{q}}^{\dagger} \hat{c}_{\mathbf{k}^{\prime}} \\
& +\hat{c}_{\mathbf{k}+\mathbf{q}}^{\dagger} \hat{c}_{\mathbf{k}} \hat{c}_{\mathbf{k}^{\prime}-\mathbf{q}}^{\dagger} \hat{c}_{\mathbf{k}^{\prime}}
\end{aligned}
$$

Inserting this last result into equation (31) one can show that:

$$
\hat{H}^{\prime}=\sum_{\mathbf{k}} E_{\mathbf{k}}^{\prime \prime} c_{\mathbf{k}}^{\dagger} c_{\mathbf{k}}+\sum_{\mathbf{k k}^{\prime} \mathbf{q}} V_{\mathbf{k k}^{\prime} \mathbf{q}} \hat{c}_{\mathbf{k}+\mathbf{q}}^{\dagger} \hat{c}_{\mathbf{k}} \hat{c}_{\mathbf{k}^{\prime}-\mathbf{q}}^{\dagger} \hat{c}_{\mathbf{k}^{\prime}}
$$

where $E_{\mathbf{k}}^{\prime \prime}$ is the re-corrected electron energy, given by:

$$
E_{\mathbf{k}}^{\prime \prime}=E_{\mathbf{k}}^{\prime}-\sum_{\mathbf{q}}\left|D_{\mathbf{q}}\right|^{2} \frac{\left(E_{\mathbf{k}+\mathbf{q}}-E_{\mathbf{k}}\right)}{\left(E_{\mathbf{k}+\mathbf{q}}-E_{\mathbf{k}}\right)^{2}-\left(\hbar \omega_{\mathbf{q}}\right)^{2}},
$$

and $V_{\mathbf{k k}^{\prime} \mathbf{q}}$ is the effective coupling resulting from the (virtual) phonon exchange, firstly derived by J. Bardeen, L.N. Cooper and J.R. Schrieffer [13], who shared the Nobel Prize in 1972 for their achievements in the theory of superconductivity, The famous result is given below $8,12,14$ :

$$
V_{\mathbf{k k}^{\prime} \mathbf{q}}=\frac{\hbar \omega_{\mathbf{q}}\left|D_{\mathbf{q}}\right|^{2}}{\left(E_{\mathbf{k}+\mathbf{q}}-E_{\mathbf{k}}\right)^{2}-\left(\hbar \omega_{\mathbf{q}}\right)^{2}} .
$$

We point out that it is usual to label $V_{\mathbf{k k}^{\prime} \mathbf{q}}$ just as $V_{\mathrm{kq}}$ in the current literature $[8,12,14]$. Notice that for $\left(\hbar \omega_{\mathbf{q}}\right)^{2}>\left(E_{\mathbf{k}+\mathbf{q}}-E_{\mathbf{k}}\right)^{2}$ the coupling $V_{\mathrm{kq}}$ becomes negative, meaning that the interaction is attractive, which is the key ingredient for 
the formation of Cooper pairs, a bound state of two electrons in momentum space, in the conventional BCS theory of superconductivity. Further discussions can be found in classical textbooks on condensed matter theory [6, 8, 12, 14].

\section{Conclusion}

In summary, in the present manuscript we presented a brief introduction to general fermion-boson hamiltonians, in which boson number is not conserved due to the fermion-boson interactions, thus complicating the search for exact solutions in closed analytical form, demanding a perturbation scheme to deal with the mathematical problem. One of the most useful methods is based on similarity transformation of the original hamiltonian, eliminating the interactions to first order in the coupling constant. A rule of thumb for obtaining the similarity transformation operator in second-quantized form is presented and applied to the problem of BCS theory of superconductivity.

\section{Acknowledgements}

C.A. Dartora would like to thank the Brazilian agency $\mathrm{CNPq}$ for partial financial support through grant and scholarship CNPq 471521/2013-2 and 301894/2014-0. The authors thank the anonymous referee for valuable contributions for the final version of this work.

\section{References}

[1] S. Weinberg, The Quantum Teory of Fields (Cambridge University Press, Cambridge, 1996), v. I and v. II, and references therein.

[2] L.H. Ryder, Quantum Field Theory, (Cambridge University Press, Cambridge, 1996), 2nd ed.

[3] M.E. Peskin and D.V. Schroeder, An Introduction To Quantum Field Theory (Addison-Wesley Publishing Company, Boston, 1995), 1st ed.

[4] E.T. Jaynes and F.W. Cummings, Proc. IEEE 51, 89 (1963).

[5] F.W. Cummings, Phys. Rev. 140, A1051 (1965).

[6] N.W. Ashcroft and N.D. Mermin, Solid State Physics (Cengage Learning, Boston, 1976), 1st ed.

[7] C. Kittel, Introduction to Solid State Physics (John Wiley and Sons, New York, 2004), 8th ed.

[8] O. Matelung, Introduction to Solid State Physics (Springer-Verlag, Berlin, 1996), 3rd ed.

[9] W. Greiner and J. Reinhardt, Field Quantization (Springer-Verlag, Berlin, 1996), 1st ed.
[10] A. Altland and B. Simons, Condensed Matter Field Theory (Cambridge University Press, Cambridge, 2010), 2nd ed.

[11] M. Tinkham, Group Theory and Quantum Mechanics (Dover Publications, New York, 2003), under permission of McGraw-Hill.

[12] C. Kittel, Quantum Theory of Solids (John Wiley and Sons, New York, 1963), 1st ed.

[13] J. Bardeen, L.N. Cooper and J.R. Schrieffer, Physical Review 108, 1175 (1957).

[14] W. Jones and N.H. March, Theoretical Solid State Physics, Volume 2, Non-Equilibrium and Disorder (Dover Edition, New York, 1985). 